\title{
Tegafur-uracil (UFT) in lower doses is safe for the treatment of colorectal cancer in patients with partial dihydropyrimidine dehydrogenase deficiency: a proof of principle
}

\author{
Daniel I. G. Cubero and Auro del Giglio
}

To the editor,

We appreciate the comments from Dr Deenen and colleagues regarding their concerns on the management of dihydropyrimidine dehydrogenase (DPD)-deficient patients [Deenen et al. 2013], in response to our article [Cubero et al. 2012]. In fact, we agree that standard-dose tegafur-uracil (UFT) is not safe as a starting dose for those patients experiencing severe toxicity after receiving 5-fluorouracil (5-FU). However, the aim of our article is to clarify that UFT can still be an option for selected patients if a careful dosing schedule is employed.

The statement that the presence of uracil in UFT creates an artificial DPD activity deficiency is a theoretical hypothesis mentioned by other authors [Niederhuber et al. 2004], including Dr Deenen in his previous case report [Deenen et al. 2010].

Interestingly, there seems to be a difference in safety profiles between 5-FU and UFT as it was demonstrated in at least two other randomized controlled trials.

In the first, Douillard and colleagues described a multicenter phase III trial comparing UFT plus leucovorin (UFT/LV) and fluorouracil plus leucovorin $(5-\mathrm{FU} / \mathrm{LV})$ in previously untreated metastatic colorectal cancer. After including 380 patients (147 patients in each arm without prior adjuvant therapy), they found that there was statistically significantly less severe leukopenia $(2 \%$ versus $12 \%$; $p<0.001)$, less severe mucositis $(2 \%$ versus $16 \%$; $p<0.001$ ) and lower rates of febrile neutropenia $(1 \%$ versus $8 \%$; $p<0.001)$ in the
$\mathrm{UFT} / \mathrm{LV}$ arm as compared with the 5-FU/LV arm [Douillard et al. 2002].

Likewise, Carmichael and colleagues presented similar results in a study including 816 patients (652 patients without previous chemotherapy), also in untreated metastatic colorectal cancer. Once again, but in a much larger sample size, there was less severe leukopenia $(<1 \%$ versus $19 \%$; $p<0.001)$, less severe mucositis $(1 \%$ versus $19 \%$; $p<0.001)$ and lower rates of febrile neutropenia ( $0 \%$ versus $13 \% ; p<0.001$ ) in UFT/LV arm in comparison with the 5-FU/LV arm [Carmichael et al. 2002].

Considering that the prevalence of partial DPD deficiency in the White population is approximately 3-5\% [Lu et al. 1993, 1998; Etienne et al. 1994], reaching up to $12.3 \%$ in specific groups such as African-American women [Mattison et al. 2006], the lower percentages of severe side effects in studies of Douillard and colleagues and Carmichael and colleagues raise the possibility that some of the patients with partial DPD deficiency are not experiencing severe toxicity.

Checking for mutations and polymorphisms in the coding region of the DPD gene is only a surrogate of DPD activity. Even patients heterozygote for some known mutations can have very low activity of DPD because there are other mechanisms involved in gene expression (epigenetic mechanisms).

In our pilot study [Cubero et al. 2012], in order to corroborate the DPD-gene sequencing, we selected only patients presenting severe toxicity
Ther Adv Med Oncol

(2013) 5(1) 93-94

DOI: $10.1177 /$

1758834012469430

(c) The Author(s), 2012. Reprints and permissions: http://www.sagepub.co.uk/ journalsPermissions.nav
Correspondence to: Daniel I.G. Cubero, MD, MSc

Department of Oncology and Haematology, ABC Foundation School of Medicine, Av. Principe de Gales, n.821, anexo 3, Santo André/SP, 09060650, Brazil

danielcuberolauol.com.br Auro del Giglio, PhD Department of Oncology and Haematology, ABC Foundation School of Medicine, Santo André, Brazil 
(grade 3 and 4) after the first cycle of 5-FU-based chemotherapy, another surrogate of DPD deficiency. The fact that not one patient developed any severe toxicity in any of the UFT cycles is a proof-of-principle that this drug is still feasible for some patients with partial DPD deficiency.

However, after a severe toxicity due to $5-\mathrm{FU}$ and a DPD-gene polymorphism is identified, if UFT is still considered, we strongly recommend an empirical reduction in UFT dose in the first cycle of chemotherapy. We do not recommend the use of UFT for those patients proven homozygote for DPD-gene mutation.

\section{References}

Carmichael, J., Popiela, T., Radstone, D., Falk, S., Borner, M., Oza, A. et al. (2002) Randomized comparative study of tegafur/uracil and oral leucovorin versus parenteral fluorouracil and leucovorin in patients with previously untreated metastatic colorectal cancer. F Clin Oncol 20: 3617-3627.

Cubero, D., Cruz, F., Santi, P., Silva, I. and Del, G. (2012) Tegafur-uracil is a safe alternative for the treatment of colorectal cancer in patients with partial dihydropyrimidine dehydrogenase deficiency: a proof of principle. Ther Adv Med Oncol 4: 167-172.

Deenen, M., Terpstra, W., Cats, A., Boot, H. and Schellens, J. (2010) Standard-dose tegafur combined with uracil is not safe treatment after severe toxicity from 5-fluorouracil or capecitabine. Ann Intern Med http://tam.sagepub.com

(SAGE journals 153: 767-768.

Deenen, M., Cats, A., Beijnen, J. and Schellens, J. (2013) Standard-dose tegafur-uracil (UFT) is not a safe alternative in partial dihydropyrimidine dehydrogenase-deficient patients Ther Adv Med Oncol 5, 91-92.

Douillard, J., Hoff, P., Skillings, J., Eisenberg, P., Davidson, N., Harper, P. et al. (2002) Multicenter phase III study of uracil/tegafur and oral leucovorin versus fluorouracil and leucovorin in patients with previously untreated metastatic colorectal cancer. f Clin Oncol 17: 3605-3616.

Etienne, M., Lagrange, J., Dassonville, O., Fleming, R., Thyss, A., Renée, N. et al. (1994) Population study of dihydropyrimidine dehydrogenase in cancer patients. f Clin Oncol 11: 2248-2253.

Lu, Z., Zhang, R., Carpenter, J. and Diasio, R. (1998) Decreased dihydropyrimidine dehydrogenase activity in a population of patients with breast cancer: implication for 5-fluorouracil-based chemotherapy. Clin Cancer Res 4: 325-329.

Lu, Z., Zhang, R. and Diasio, R. (1993)

Dihydropyrimidine dehydrogenase activity in human peripheral blood mononuclear cells and liver: population characteristics, newly identified deficient patients, and clinical implication in 5-fluorouracil chemotherapy. Cancer Res 53: 5433-5438.

Mattison, L. K., Ezzeldin, H., Carpenter, M., Modak, A., Johnson, M.R. and Diasio, R.B. (2006) Increased prevalence of dihydropyrimidine dehydrogenase deficiency in African-Americans compared with Caucasians. Clin Cancer Res 12: 5491-5495.

Niederhuber, J., Cole, C. and Grochow, L. (2004) Colon cancer. In Abeloff, M., Armitage, J., Niederhuber, J., Kastan, M. and McKenna, W. (eds), Clinical Oncology, 3rd edition. Philadelphia, PA: Elsevier Churchill Livingstone, 2004; pp. 1928-1929. 